Applied Physiology, Nutrition, and Metabolism

Canadian Science Publishing Physiologie appliquée, nutrition et métabolisme

\title{
Heterogeneous Effects of Old Age on Human Muscle Oxidative Capacity in Vivo: A Systematic Review and Meta- Analysis
}

\begin{tabular}{|r|l|}
\hline Journal: & Applied Physiology, Nutrition, and Metabolism \\
\hline Manuscript ID & apnm-2016-0195.R1 \\
\hline Manuscript Type: & Article \\
\hline Date Submitted by the Author: & 26 -Jun-2016 \\
\hline Complete List of Authors: & $\begin{array}{l}\text { Fitzgerald, Liam; University of Massachusetts Amherst } \\
\text { Christie, Anita; University of Oregon } \\
\text { Kent, Jane; University of Massachusetts Amherst, Kinesiology Department }\end{array}$ \\
\hline Keyword: & $\begin{array}{l}\text { mitochondria, oxidative phosphorylation, bioenergetics, aging, magnetic } \\
\text { resonance spectroscopy }\end{array}$ \\
\hline
\end{tabular}

\section{SCHOLARONE}

Manuscripts 


\title{
Heterogeneous Effects of Old Age on Human Muscle Oxidative Capacity in Vivo: A
} Systematic Review and Meta-Analysis

\author{
Liam F. Fitzgerald, ${ }^{1}$ Anita D. Christie, ${ }^{2}$ and Jane A. Kent ${ }^{1}$ \\ ${ }^{1}$ Department of Kinesiology, University of Massachusetts Amherst, MA, USA \\ ${ }^{2}$ Department of Human Physiology, University of Oregon, Eugene, OR, USA
}

Liam F. Fitzgerald, M.S, Department of Kinesiology, 110 Totman Building, 30 Eastman

Lane, University of Massachusetts, Amherst, MA, 01003, USA

Email: 1ffitzgerald@kin.umass.edu

Anita D. Christie, PhD, Department of Human Physiology, 342 Gerlinger, 122 Esslinger

Hall, 1240 University of Oregon, Eugene, OR, 97403, USA

Email: adc1@uoregon.edu

Corresponding Author:

Jane A. Kent, PhD, Department of Kinesiology, 160A Totman Building, 30 Eastman Lane, University of Massachusetts, Amherst, MA, 01003, USA

Tel: (413) 545-9477

Fax: (413) 545-2906

Email:jkent@kin.umass.edu 


\begin{abstract}
Despite intensive efforts to understand the extent to which skeletal muscle mitochondrial capacity changes in older humans, the answer to this important question remains unclear. To determine what the preponderance of evidence from in vivo studies suggests, we conducted a systematic review and meta-analysis of the effects of age on muscle oxidative capacity as measured noninvasively by magnetic resonance spectroscopy. A secondary aim was to examine potential moderators contributing to differences in results across studies, including: muscle group, physical activity status, and sex. Candidate papers were identified from PubMed searches ( $n=3,561$ papers) and the reference lists of relevant papers. Standardized effects (Hedges' $g$ ) were calculated for age and each moderator using data from the 22 studies that met the inclusion criteria ( $\mathrm{n}=28$ effects). Effects were coded as positive when older $(\geq 55$ years) adults had higher muscle oxidative capacity than younger (20-45 years) adults. The overall effect of age on oxidative capacity was positive ( $\mathrm{g}=0.171, \mathrm{p}<0.001)$, indicating modestly greater oxidative capacity in old. Notably, there was significant heterogeneity in this result $\left(\mathrm{Q}=245.8, \mathrm{p}<0.001 ; \mathrm{I}^{2} \sim 70-90 \%\right)$. Muscle group, physical activity, and sex were all significant moderators of oxidative capacity $(\mathrm{p} \leq 0.029)$. This analysis indicates that the current body of literature does not support a de facto decrease of in vivo muscle oxidative capacity in old age. The heterogeneity of study results and identification of significant moderators provide clarity regarding apparent discrepancies in the literature, and indicate the importance of accounting for these variables when examining purported age-related differences in muscle oxidative capacity.
\end{abstract}

Key words: mitochondria, oxidative phosphorylation, oxygen, bioenergetics, aging, 31phosphorus magnetic resonance spectroscopy, aerobic 


\section{INTRODUCTION}

Muscle oxidative capacity can be defined in vivo as the maximal rate at which a muscle can consume oxygen under conditions in which delivery of oxygen and substrate are not limited (Chance et al. 1985). This rate thus reflects the maximal rate of oxidative phosphorylation in the volume of mitochondria present in the tissue. Currently, it is not clear whether muscle oxidative capacity declines in old age, as some investigators have reported lower oxidative capacity in older than younger individuals (Conley et al. 2000; Johannsen et al. 2011; Layec et al. 2013; McCully et al. 1993; Smith et al. 1998; Taylor et al. 1997), while others found no difference (Chilibeck et al. 1998; Christie et al. 2014; Hart et al. 2014; Horská et al. 2000; Kent-Braun and Ng 2000; Kutsuzawa et al. 2001; Lanza et al. 2005; Lanza et al. 2007; Larsen et al. 2012; Layec et al. 2015; Taylor et al. 1984; Tevald et al. 2014; Tevald et al. 2010; Wray et al. 2009) or even a higher capacity in old compared with young (Larsen et al. 2012; Tevald et al. 2014). It seems that at least a portion of this discrepancy may be due to differences in potential modifiers of oxidative capacity across studies, such as muscle group, habitual physical activity (PA) status, and sex.

Since the 1980s, researchers have probed the oxidative capacity of muscle in vivo by quantifying oxidative ATP production using 31-phosphorus magnetic resonance spectroscopy (MRS). This technique detects the phosphorus signal from an identified volume of interest in the muscle that contains several distinct peaks based on the resonant frequency of each phosphorus-containing compound (e.g., phosphocreatine (PCr), inorganic phosphate $\left(\mathrm{P}_{i}\right)$, ATP). The area under each peak indicates the relative concentration of each compound, which can be monitored continuously, noninvasively, and repeatedly. Following a brief contraction in which intracellular $\mathrm{pH}$ is relatively unaltered, the kinetics of $\mathrm{PCr}$ recovery follow a mono-exponential curve that is strictly attributable to mitochondrial ATP production (Arnold et al. 1984; Meyer 1988; Paganini et al. 1997; Quistorff et al. 1993). From this 
curve, the rate constant of $\mathrm{PCr}$ recovery $\left(k_{\mathrm{PCr}}, \mathrm{s}^{-1}\right)$ can be determined and used as an index of muscle oxidative capacity (Kent-Braun et al. 1995); this measure has become the "gold standard" for evaluating muscle oxidative capacity in vivo. Notably, oxidative capacity measured in this way using ${ }^{31} \mathrm{P}-\mathrm{MRS}$ is highly reliable and well correlated with respirometry measures of mitochondrial capacity (Lanza et al. 2011) and oxidative enzyme activities (Larson-Meyer et al. 2001; McCully et al. 1993).

One of the key benefits of investigating muscle oxidative capacity using ${ }^{31} \mathrm{P}-\mathrm{MRS}$ is the non-invasive and continuous nature of the data collected. However, it is important to note that some research questions will require an in vitro approach. For example, while ${ }^{31} \mathrm{P}-\mathrm{MRS}$ measures can yield an indirect measure of a muscle's oxidative capacity, it cannot be used to determine mitochondrial content or potential molecular mechanisms of altered oxidative capacity with age. Further, ${ }^{31} \mathrm{P}-\mathrm{MRS}$ only detects phosphorus-containing compounds within the cytosol, and so cannot be used to evaluate, for example, whether aging affects $\mathrm{PCr}$ shuttling of ATP from the mitochondria to the cytosol (Sahlin and Harris 2011; Walsh et al. 2001). Likewise, it is important to acknowledge that mitochondria serve many functions in the cell, including energy production, reactive oxygen species signalling, and intrinsic pathways of apoptosis (e.g., mitophagy) (Hepple 2014). It is possible that all, some, or none of these functions change with age and could contribute to alterations in in vivo oxidative capacity as measured by ${ }^{31} \mathrm{P}$ MRS.

The discrepant findings regarding age-related changes in muscle oxidative capacity to date are likely confounded by variations among study cohorts in age, muscle group(s) under study, sex, PA, health status, and muscle contraction protocols (e.g., relative intensity). Indeed, a review on this topic published in 2004 concluded that future studies should account for the health status and PA of their participants (Russ and Kent-Braun 2004), and to some extent this has occurred. Because PA is well documented to decrease from adulthood into old 
age (Colley et al. 2011; Troiano et al. 2008), accounting for its effects in studies of agerelated changes in muscle oxidative capacity is essential. Likewise, results from recent studies indicate that different muscles, particularly those involved in locomotion, undergo different age-related changes in habitual loading patterns during movement; these differences have been suggested to explain a portion of the muscle-specific changes in oxidative capacity reported in old age (Larsen et al. 2012). Further, sex-based differences in muscle oxidative capacity have been reported in the literature (Kent-Braun and Ng 2000; Waters et al. 2003), but this variable is often not accounted for in studies designed to quantify the effects of old age on muscle oxidative capacity. Finally, additional factors such as health status can complicate the interpretation of results in studies of muscle mitochondrial and oxidative function.

Therefore, the purpose of this systematic review and meta-analysis was to obtain a comprehensive, quantitative evaluation of the published literature on in vivo muscle oxidative capacity and aging. We hypothesized that oxidative capacity would be moderated by muscle group, PA, and sex. To examine the quality of the studies included in our analysis, we also evaluated the magnitude of the contraction-induced changes in $\mathrm{PCr}$ and intracellular $\mathrm{pH}$. Our analysis provides quantitative information regarding the pertinent characteristics moderating muscle oxidative capacity in older adults.

\section{METHODS}

\section{PCr Recovery Method}

This systematic review was restricted to studies in which muscle oxidative capacity was quantified using the MRS PCr recovery method (Marsh et al. 1993; Meyer 1988). This approach has the advantage of limiting the influence of methodological approach on study outcomes, and focusing the review on age-related changes in oxidative capacity in vivo. Optimal conditions for this measure include: full activation of the muscle volume of interest, 
depletion during contraction of $[\mathrm{PCr}]$ by $\approx 50 \%$ from baseline, and minimal change in intracellular pH (Arnold et al. 1984; Meyer 1988). Under these conditions, the postcontraction recovery of $\mathrm{PCr}$ is dependent on oxidative phosphorylation and follows a monoexponential time course. In addition to the rate constant $\left(k_{\mathrm{PCr}}, \mathrm{s}^{-1}\right)$, other variables derived from the mono-exponential fit of $\mathrm{PCr}$ recovery include: tau $\left(1 / k_{\mathrm{PCr}}, \mathrm{s}\right), \mathrm{t}_{1 / 2}$ (time to recover PCr to $50 \%$ of baseline, $\mathrm{s}$ ), and $\mathrm{V}_{\max }$ (an estimate of the muscle's maximal rate, calculated as $k_{\mathrm{PCr} \times}($ resting $\left.[\mathrm{PCr}]), \mathrm{mM} \cdot \mathrm{s}^{-1}\right)$. All of these variables provide robust indices of muscle oxidative capacity (Lanza et al. 2011; Larson-Meyer et al. 2000; Layec et al. 2009) and are well-correlated with respirometry measures of mitochondrial capacity (Lanza et al. 2011) and oxidative enzyme activities (Larson-Meyer et al. 2001; McCully et al. 1993).

\section{Literature Search}

The analysis was performed in accordance with the Meta-Analysis of Observational Studies (Stroup et al. 2000) recommendations. All searches were performed by the same investigator (L.F.F.) following instruction from a qualified librarian and with guidance from the co-investigators. Final searches were carried out on 8 September 2015. The literature search was conducted using the PubMed online database, with combinations of the following keywords and MeSH headings: "mitochondria OR mitochondrial," "oxidative capacity OR oxidative phosphorylation," "ageing OR aging," "nuclear magnetic resonance OR NMR OR magnetic resonance spectroscopy OR MRS OR magnetic resonance," “elderly OR older," "skeletal muscle," "physical activity," "creatine kinase OR phosphocreatine recovery OR adenosine triphosphate OR ATP," "bioenergetics," "metabolism," and "aerobic metabolism." The search returned 3,561 articles and studies were included if they met all of the following criteria: $i$ ) involved a direct comparison of young and older humans, ii) used ${ }^{31} \mathrm{P}$ MRS measures of PCr recovery kinetics for calculating oxidative capacity of skeletal muscle, iii) original research articles, $i v$ ) written in English, and $v$ ) included data necessary for calculating 
standardized effects (effects, Hedges' g). All studies involved cross-sectional comparisons by age; we defined 'young' as 20-45 years and 'older' as $\geq 55$ years, based on the group means used in the papers included for review.

Beginning with the 3,561 studies returned by our searches, papers were removed for the following reasons: 3,403 studies were removed because they did not include a direct comparison of young and older humans, 52 did not include a measure of oxidative capacity by $\mathrm{PCr}$ recovery kinetics, 43 were not original research articles, 28 were case studies, 3 were not written in English, 1 was not conducted in skeletal muscle, in 1 paper the statistics could not be converted to a standardized mean difference, and 1 paper had been retracted. A further 10 studies were removed because 1 or more search returned the same study (i.e., duplicates). An additional 2 studies were removed because data were reported on the same participants as in an earlier paper by the same authors that was already included in our analysis. From the remaining 17 studies, authors were contacted if their results appeared to be from the same participants (e.g., similar group mean and variance for cohorts), and 1 additional study was removed. One study reported calculations for PCr recovery kinetics, but no data were presented. In this case, the corresponding author was contacted and the relevant results were kindly provided. The reference lists of the final 16 studies were manually searched for inclusion in our analyses, and an additional 6 studies were identified, bringing the total number of studies to 22 . Some $(n=4)$ of the included studies measured oxidative capacity in more than one muscle (e.g., KE and DF), or more than one cohort (e.g., physically active vs. inactive). Accordingly, these studies have more than 1 effect size, bringing the total number of standardized effects (effects) to 28. None of the included studies were conducted in diseased populations. 


\section{Calculation of Standardized Effects}

The standardized difference in means (effect) provides an index of the magnitude of an effect (Hedges 1984). In the present analyses, effects were calculated as the difference in group means, divided by the pooled standard deviation (SD), using the Comprehensive MetaAnalysis software, Version 3.3 (Biostat, Englewood, NJ), as follows:

$$
\left(\bar{X}_{\text {young }}-\bar{X}_{\text {older }}\right) / S D_{\text {Pooled }}
$$

A fixed effects model was used to assess effects across studies, assuming samples were drawn from different populations and that there was one true effect. This model weighted each effect based on the sample size in each study. The fixed effects model was used as it directly accounts for the sample size in each study, and sample sizes are typically small in ${ }^{31} \mathrm{P}$ MRS experiments.

In the present analyses, we selected indices of oxidative capacity in the following order: 1) $k_{\mathrm{PCr}}\left(\mathrm{s}^{-1}\right)$, 2) tau or $\mathrm{t}_{1 / 2}(\mathrm{~s})$, and 3) $\mathrm{V}_{\max }\left(\mathrm{mM} \mathrm{ATP.s}{ }^{-1}\right)$. This order was chosen to limit the number of biochemical assumptions made to obtain the variable (i.e., $V_{\max }$ was used only if necessary, as additional assumptions are required for that calculation). In studies that had 2 exercise bouts $(n=2)$ we chose either the first exercise bout if both bouts were of the same intensity, or the more intense exercise bout if one bout was performed at a higher intensity, as the latter condition was assumed to activate a greater portion of the muscle mass under study. In the one study where values from the more intense exercise bout were used, there was no difference in end-contraction $\mathrm{pH}$ between young and older groups $(\mathrm{p}=0.31$, based on $t$ test using reported data). Data were coded such that a positive effect indicated greater oxidative capacity in older compared with younger adults. 


\section{Evaluation of Bias}

Publication bias was assessed through the construction of a funnel plot of effects versus standard errors (SE). Asymmetries or gaps in a funnel plot suggest the presence of a publication bias (Christie et al. 2011; Egger et al. 1997). Also, potential bias was accounted for quantitatively in the calculation of the overall effect and the effect for each moderator by weighting the effects on the basis of their SE.

\section{Calculation of Heterogeneity}

If the effect of age on oxidative capacity was the same for all 22 studies included here, then the effect would be entirely homogenous, regardless of the direction of the result. However, if the effect of age on oxidative capacity differs across studies, the effect becomes heterogeneous (Sutton and Higgins 2008). In our analyses, we evaluated the heterogeneity of effects using the $95 \%$ confidence interval (CI) around the mean effect, and by calculating the $\mathrm{Q}$ and $\mathrm{I}^{2}$ statistics, using Comprehensive Meta-Analysis. The $\mathrm{Q}$ statistic is calculated as the sum of squares of the effect for each study, divided by the overall mean effect. A statistically significant Q indicates heterogeneous standardized effects. The $\mathrm{I}^{2}$ statistic describes the percentage of total variation across studies due to heterogeneity, and is calculated as follows:

$$
I^{2}=100 \% \times((Q-d f) / Q)
$$

Where Q and $d f$ represent the Q statistic and degrees of freedom, respectively. Negative values of $\mathrm{I}^{2}$ are set equal to zero, so that interpretation of $\mathrm{I}^{2}$ lies between $0-100$ percent, where a larger value represents greater heterogeneity (Higgins et al. 2003).

\section{Moderator Coding}

Moderators are factors that have been suggested to contribute in a meaningful way to age-related differences in muscle oxidative capacity. For the purpose of this analysis, moderators were selected based on those commonly included in the discussion sections of the 
22 studies evaluated here, as well as in a previous review paper (Russ and Kent-Braun 2004).

All moderators in this analysis were coded as categorical variables, in line with MOOSE recommendations (Stroup et al. 2000). The following moderators (and categories within each moderator) were identified for this study: muscle group (dorsiflexors, DF; plantar flexors, PF; knee extensors, KE; upper extremity, UE), sex (women, men, or mixed groups), and PA status (low, moderate, or high). To evaluate the potential influence of variations in experimental conditions, PCr depletion (\% resting; young $>$ older, young $<$ older, or no difference, as reported in each study) and $\mathrm{pH}$ (young $<$ older, young $>$ older, or no difference, as reported in each study) following the contraction protocol were also examined as potential moderators. One study was not included in the sex analyses because their young group was all males and the older group contained one female. However, this study was included in all other analyses. Physical activity status was coded based on the classifications used in the original article, and only where PA was measured by questionnaire or accelerometry. Only 1 study examined the forearm flexors, and 3 studies examined finger flexors (e.g., flexor digitorum superficialis). Unlike the muscles of the lower extremity, neither of these muscle groups is involved in ambulation, and as such these four studies were grouped together as 'UE' in our analyses.

\section{RESULTS}

Twenty-two studies were included in the present analysis. The mean ages, number of effects, and number of studies for each direction (i.e., positive or negative effect in old compared with young) for all studies are presented in Table 1. Fourteen effects (from 12 studies) indicated greater muscle oxidative capacity in older compared with young muscle, 13 effects (from 12 studies) indicated lower muscle oxidative capacity in the older muscle, and 1 effect indicated no difference in muscle oxidative capacity between young and older muscle (Table 1). 
In 4 of the included studies, no participants were taking any medications (Smith et al. 1998; Taylor et al. 1984; Taylor et al. 1997; Wray et al. 2009). In 4 of the included studies, the number and type of medications that older adults were taking was not specifically addressed (Chilibeck et al. 1998; Horská et al. 2000; Kutsuzawa et al. 2001; Waters et al. 2003). In 10 of the included studies, no participants were taking any medications known to affect muscle or metabolic function (Christie et al. 2014; Hart et al. 2014; Johannsen et al. 2011; Kent-Braun and Ng 2000; Lanza et al. 2005; Lanza et al. 2007; Larsen et al. 2012; Layec et al. 2015; Layec et al. 2013; Tevald et al. 2010). In 1 study, 9 out of 22 older women were receiving hormone replacement therapy (Conley et al. 2000). In another study, 17 out of 27 older adults were taking non-steroidal anti-inflammatory drugs for chronic low-back pain (Mattei et al. 1999). One study reported 1 older adult to have a history of myocardial infarction, but medications for this individual were not reported (McCully et al. 1993). Lastly, in one study, subjects took angiotensin-converting enzyme inhibitors $(n=3)$, angiotensin receptor blockers $(n=1)$, diuretics $(n=2)$, statins $(n=4)$, and hormone replacement therapy $(\mathrm{n}=1)$ (Tevald et al. 2014).

\section{Standardized Effects}

A total of 28 effects were analyzed from 22 studies; these are grouped by muscle and summarized in Table 2. The overall effect of age on muscle oxidative capacity was positive and significant (Table 2), indicating modestly greater oxidative capacity in older compared with young muscle.

\section{Heterogeneity and Bias}

Our analyses indicate significant heterogeneity regarding age-related differences in muscle oxidative capacity across studies $\left(\mathrm{Q}=245.8, \mathrm{I}^{2}=89 \%, \mathrm{p}<0.001\right)$. The funnel plot (Figure 1) illustrates the SE plotted against each effect (Hedges' $g$ ). Because the majority of the open circles are located within the two angled lines, and the number of open circles are 
evenly distributed about the center line representing the overall mean effect, this plot indicates a lack of substantial publication bias. It does appear that there is one outlier, with an effect of -7.65 , but this does not change the interpretation of the funnel plot.

\section{Moderators of the Effects of Age on Oxidative Capacity}

To evaluate how the moderators influenced the relationship between age and oxidative capacity, each moderator was examined with the use of sub-group analysis. The number of effects for the sub-groups of each moderator are presented in Table 3, along with the effect (Hedges' g), 95\% CI, p-value for the Q-statistic, and $\mathrm{I}^{2}$ statistic. The Q-statistic was significant for the sub-groups of each moderator, with the exceptions of moderate PA, and younger adults having a higher $\mathrm{pH}$ than older adults at the end of the contraction protocol. However, of the 28 effects included in this analysis, only one study found $\mathrm{pH}$ at the end of contraction to be higher in younger adults compared with older adults. Therefore, the Q- and $\mathrm{I}^{2}$ statistics cannot be interpreted for this sub-group of the moderator. The significance of the Q-statistic for the majority of moderator sub-groups indicates high heterogeneity across studies, which is also reflected in the $\mathrm{I}^{2}$ values shown in Table 3.

Muscle Group. The overall and sub-group effects for muscle group as a moderator are shown in Table 2. The effect was positive for UE $(\mathrm{p}=0.01)$ and $\mathrm{DF}(\mathrm{p}<0.001)$, but negative for $\mathrm{KE}(\mathrm{p}<0.001)$ and $\mathrm{PF}(\mathrm{p}=0.492)$; overall sub-group effects were significant only for $\mathrm{KE}$, UE, and DF. The varying direction of the effects across muscle groups indicates that oxidative capacity is influenced by age differently, depending on the muscle under study. The effect and 95\% CI for each muscle group is illustrated in Figure 2, showing the heterogeneity of effects across studies.

Physical Activity. The effects for PA were positive across the 3 sub-groups used for categorization of studies: low, moderate, and high. However, the effect was modest for low PA $(p=0.003)$ and non-significant for moderate $(p=0.693)$ and high PA $(p=0.956)$, as 
illustrated in Figure 2. The distribution of effects between low, moderate, and high indicates that the majority of studies examined participants with low PA. Nine effects from 9 studies did not measure or report an accelerometry or questionnaire measure of PA.

Sex. For the moderator analyses, sex was categorically coded as male, female, or mixed groups for each study. These results are summarized in Table 3. The effect was positive for males $(\mathrm{p}=0.002)$ and the mixed groups $(\mathrm{p}=0.008)$ analyses, but negative in the female group $(\mathrm{p}=0.001)$.

Study Conditions: $\mathrm{PCr}$ and $\mathrm{pH}$. Sub-groups for end-contraction $\mathrm{PCr}$ level were coded as no difference in PCr depletion between old and young, a greater PCr depletion in old compared with young, or a lower PCr depletion in old compared with young. The number and magnitude of effects for each sub-group is shown in Table 3. Importantly, most studies ( $\mathrm{n}=14$ effects) observed no difference in PCr depletion between older and young adults, indicating that these studies met the criterion of similar PCr depletion between groups.

Similarly, sub-groups for end-contraction $\mathrm{pH}$ were coded as no difference between old and young, higher $\mathrm{pH}$ in old, or lower $\mathrm{pH}$ in old. Only one study reported a lower $\mathrm{pH}$ in old muscle following the contraction protocol; this study also had the largest effect of age on muscle oxidative capacity. Importantly, the majority of studies ( $\mathrm{n}=15$ effects) reported no difference in end-contraction $\mathrm{pH}$ across age groups, again indicating that these studies were generally well-designed.

\section{DISCUSSION}

The purpose of this study was to provide a quantitative analysis of the existing literature regarding age-related changes in muscle oxidative capacity in vivo. The results of this systematic review and meta-analysis indicate that, while the overall effect of age on skeletal muscle oxidative capacity is positive, indicating greater oxidative capacity in older muscle in vivo, there is significant heterogeneity across studies in this result. Closer 
examination of the moderators suggested to mediate this effect indicates that muscle oxidative capacity changes with age depending upon muscle group, PA, and sex.

Muscle Group. Our results indicate that muscle group significantly moderates the effect of age on oxidative capacity (Tables 2 and 3, Figure 2). Specifically, UE and DF exhibited greater oxidative capacity in older muscle, while KE and PF muscles had lower oxidative capacity in older compared with young. These results provide strong new evidence that the current body of literature supports the hypothesis that the effects of age vary by muscle. It is likely that this difference across muscles is, in part, due to age-related differences in PA patterns, as suggested by Larsen et al. (2012). It has also been suggested (Coggan et al. 1990) that the lower maximal whole-body aerobic capacity of older adults, which results in day-to-day activities being performed at a higher relative workload, may be sufficient to result in training-like adaptations in some but not all muscles. Further, gait mechanics have been shown to change with age, such that the total support torque is achieved via different contributions in young compared with older adults. Specifically, with age the KEs and PFs contribute less and the hip extensors contribute more of the total support torque during gait (Boyer et al. 2012; DeVita and Hortobagyi 2000).

Generally, our analyses demonstrate a modestly increased muscle oxidative capacity in vivo in older adults. However, there is significant heterogeneity in this result, largely due to variations in the muscle studied. During locomotion, the tibialis anterior is recruited to a similar extent regardless of walking or running speed; conversely, recruitment of the vastus lateralis increases with speed (Cappellini et al. 2006). As such, it has been suggested that higher intensity activities might be needed to preserve oxidative capacity in the KE; indeed, in vivo oxidative capacity in the vastus lateralis of older adults is significantly associated with daily minutes of moderate-to-vigorous PA (Larsen et al. 2012). In contrast, DF oxidative 
capacity may be preserved the by usual locomotory activities of healthy, community-dwelling older adults.

Five effects were reported for the KE, all of which were negative (see Table 2). While all 5 studies indicated lower muscle oxidative capacity in the old, there was significant heterogeneity in this result $\left(\mathrm{I}^{2}=0.94 \%\right.$, Table 3$)$, reflecting the large range of effect sizes across the 5 studies. It is likely that this range in effects for the difference in KE oxidative capacity with age is due to variations in participant characteristics, such as sex and PA, across studies. Further research is needed to address the potential mechanisms by which in vivo oxidative capacity is reduced in the KE muscles of older adults.

Eleven effects were reported for the PF, which consist of the soleus and both medial and lateral heads of the gastrocnemii. Given that these muscles have varying architecture, fiber-type composition, and functional roles, it is reasonable to anticipate that a portion of the significant heterogeneity of results in this muscle group could have been due to sampling different portions of the PF. Future studies that systematically examine the muscles important for ambulation and balance, as well as the moderating effects of PA on these muscles, are needed.

Physical Activity. Of the 22 studies (28 effects) included in our overall analysis, only 13 studies (19 effects) included a measure of PA, and of those, 10 studies (13 effects) used objective measures of PA (i.e., accelerometry). Each of the sub-groups of PA (low, moderate, and high) showed a positive effect that varied widely in magnitude (Table 3, Figure 2). It is notable that the sub-group of studies reporting low PA were nearly evenly split in terms of the effect of age on muscle oxidative capacity (Table 1). The paucity of studies that included cohorts with moderate and high PA render interpretation of these sub-groups difficult. 
Overall, these results confirm that it is imperative to account for PA when investigating the primary effects of age on human skeletal muscle oxidative capacity. In our analysis, we included studies that accounted for PA by use of questionnaire or accelerometer. Because questionnaire measures of PA are susceptible to recall bias and social desirability (Strath et al. 2013), objective measures of PA (e.g., accelerometry) are the preferred measure for quantifying PA volume and intensity. Finally, a potentially fruitful avenue for future research includes exercise interventions that are designed to determine the magnitude of exercise required to elicit a meaningful increase in muscle oxidative capacity in older adults, that is, an increase sufficient to produce clinically-relevant improvements in energy metabolism and mobility.

Sex. The effects for male and mixed groups of participants were positive, but studies with only females yielded a negative effect, indicating higher oxidative capacity in older compared with young males, and in young compared with older females (Table 3). Along with this apparent age-by-sex interaction, it should be noted that there was significant heterogeneity across studies for each sex sub-group (Figure 2). It is important to acknowledge that the mixed sex sub-group had a considerably larger number of effects $(n=18)$ than male $(n=6)$ or female $(n=3)$ only groups, again making definitive interpretations difficult. Future studies examining muscle oxidative capacity with age should include both sexes with sufficient sample sizes to elucidate the potential confounding factors driving the apparent interactions between age, sex, and muscle oxidative capacity observed in this study.

Study Conditions: $\mathrm{PCr}$ and $\mathrm{pH}$. To gain meaningful insight into $\mathrm{PCr}$ recovery kinetics, $\mathrm{PCr}$ at the end of the contraction protocol needs to be sufficiently depleted and comparable across study groups. A total of 20 effects from 19 studies reported the percentage of PCr depletion during exercise. Of those, 14 effects showed no difference between young and older adults, 3 effects indicated greater PCr depletion in older adults, and 3 effects 
indicated that younger adults depleted PCr more than older adults; in all cases, there remained a positive effect for oxidative capacity, such that older had a greater capacity than young (Table 3). Likewise, it is important to note the extent of $\mathrm{PCr}$ depletion for the studies included in our analyses were within the $20-70 \%$ range appropriate for application of Meyer's linear respiration model for evaluating in vivo oxidative capacity (Meyer 1988).

Another important methodological concern in ${ }^{31} \mathrm{P}-\mathrm{MRS}$ experiments is the degree of acidification during and following the contraction protocol, as significant acidosis will slow the rate of oxidative phosphorylation and thus PCr recovery (Arnold et al. 1984). As long as the change in $\mathrm{pH}$ is small, the resynthesis of $\mathrm{PCr}$ is attributable strictly to mitochondrial ATP production (Arnold et al. 1984; Meyer 1988; Paganini et al. 1997; Quistorff et al. 1993) and follows a mono-exponential time course. In our analysis, 15 effects from 14 studies formed a sub-group with no difference in $\mathrm{pH}$ at the end of contraction between age groups (Table 3). Only 1 effect indicated a lower $\mathrm{pH}$ in older muscle at the end of contraction, and the difference in $\mathrm{pH}$ between groups was modest (7.02 vs. 7.04 units). Conversely, 7 effects from 7 studies found younger muscle to have a lower $\mathrm{pH}$ at the end of contraction than older muscle. Of the 22 studies included in our analysis, only 1 study reported a large difference in $\mathrm{pH}$ (0.5 units), and this did not differ between young and older muscle. Overall, the changes in $\mathrm{PCr}$ and $\mathrm{pH}$ induced by the various contraction protocols indicate comparable metabolic conditions in the muscles of young and older adults. These results support the notion that the studies included in our analysis were generally well-conducted and met the criteria for quantifying in vivo muscle oxidative capacity using the PCr recovery method.

\section{Additional Factors}

In this systematic review and meta-analysis, we quantified the contributions to agerelated differences in muscle oxidative capacity by the most commonly-suggested moderators of this relationship. We acknowledge that some of these moderators may interact with each 
other to further influence the relationship between age and oxidative capacity, but clarification of these potential interactions will require studies designed with that goal in mind. For example, the finding of a significant sex effect is currently based on only a few studies; a more complete evaluation of muscle oxidative capacity in men and women is needed.

Factors other than those considered here also may moderate the relationship between muscle oxidative capacity and age. For example, some authors have reported that muscle perfusion capacity limits its oxidative capacity (Haseler et al. 1999). There are discrepancies in this result such that insufficient oxygen delivery can limit muscle oxidative capacity in highly-trained individuals, but this is not the case in sedentary individuals (Hart et al. 2014; Haseler et al. 2004). To date, there is a lack of sufficient data examining changes in perfusion and oxidative capacity of muscle in aging to permit a formal analysis of perfusion as a moderator of age-related changes in muscle oxidative capacity in vivo. Likewise, muscle fiber composition may change toward a slower phenotype with age (Jakobsson et al. 1990; Lexell et al. 1988), habitual PA decreases across the lifespan (Troiano et al. 2008), and aerobic fitness is lower in old age (Proctor and Joyner 1997). As such, these factors, and others, may impact age-related changes in muscle oxidative capacity. To date, the dearth of research examining these variables in addition to measures of oxidative capacity in vivo precluded a formal statistical analysis of their moderating effects in this systematic review and meta-analysis.

Two additional knowledge gaps have been identified by our study. First, to date, few studies have quantified muscle oxidative capacity in very old ( $>80$ years) individuals, nor have chronological studies of in vivo oxidative capacity been performed in the aged. Second, the extent to which the moderators of oxidative capacity identified here influence in vitro measures of oxidative and mitochondrial function remains to be fully determined. 
Finally, the observation that, overall, muscle oxidative capacity is maintained or even enhanced in older adults when measured in vivo by ${ }^{31} \mathrm{P}-\mathrm{MRS}$ is in contrast with some studies reporting deleterious effects of old age on mitochondrial function in vitro (Short et al. 2005; Tonkonogi et al. 2003). It should be noted that, when mitochondrial function is measured in isolated mitochondria, the organelle is susceptible to structural disruption (Hepple 2014; Picard et al. 2011b), which can exaggerate the magnitude of age-related impairments in mitochondrial function compared with saponin-permeabilized fiber bundles (Picard et al. 2011c). This latter in vitro approach does not disrupt organelle structure (Saks et al. 1998). In fact, some studies using permeabilized myofiber bundles from human vastus lateralis muscle have found no effect of age on maximal mitochondrial respiratory capacity (Gouspillou et al. 2014; Hütter et al. 2007). Interestingly, one such study reported a muscledependent decrease in state III respiration with age in rats, such that muscles with a slower phenotype exhibit a reduced oxidative capacity with age and muscle with a faster phenotype demonstrate a propensity for preservation with age (Picard et al. 2011a). Until recently, most in vitro studies of mitochondrial function in aging have not accounted for differences in PA of the study cohort, and so it is reasonable to expect that PA may have influenced those results. While ${ }^{31} \mathrm{P}-\mathrm{MRS}$ has the advantage of quantifying oxidative capacity non-invasively in the intact system, at this time an understanding of the mechanistic basis of age-related changes in oxidative capacity requires an in vitro approach. The results of this systematic review and meta-analysis suggest a need to target future mechanistic studies on muscles that demonstrate age-related decreases in oxidative capacity in vivo (e.g., the knee extensors), while accounting for sex and PA habits. Moreover, a combined approach of in vivo and in vitro methods, with adequate control for potential modifiers of oxidative capacity such as those identified here, may prove optimal. 


\section{Conclusions}

This systematic review and meta-analysis provides the first quantitative evaluation of the literature regarding the overall impact of age on muscle oxidative capacity, as well as insight as to the variables that moderate the relationship between muscle oxidative capacity and age. The results from these cross-sectional studies indicate, overall, a modestly greater oxidative capacity in older compared with young muscle. Perhaps more importantly, it is clear that the muscle under study is critical to this outcome: oxidative capacity is lower in the $\mathrm{KE}$ of older adults, similar between young and older adults in the PF, and greater in the UE and DF of older compared with younger adults. The higher oxidative capacity in DF of older muscle appears to correspond with its daily, submaximal use in mobile adults. In contrast, oxidative capacity in the more power-oriented KE muscles, which are most often the muscles biopsied for in vitro studies of mitochondrial function, show markedly lower oxidative capacity. The observation of significant moderating effects by PA and sex, despite relatively few studies that addressed these moderators, indicates a need for additional work in order to clarify the conditions and extent to which each moderator is important. Thus, the results of this meta-analysis reconcile and clarify the existing literature with regard to muscle oxidative capacity. We conclude that the body of evidence at this time indicates no de facto decrease in human skeletal muscle oxidative capacity in vivo. 
Conflict of Interest Statement. The authors declare that there are no conflicts of interest.

Acknowledgements. The authors thank the members of the Muscle Physiology Lab for their helpful input to this project, and the researchers who provided additional details about their data for these analyses.

Funding. L.F.F. was supported by a School of Public Health \& Health Sciences Dean's Doctoral Student Fellowship. 


\section{REFERENCES}

Arnold, D.L., Matthews, P.M., and Radda, G.K. 1984. Metabolic recovery after exercise and the assessment of mitochondrial function in vivo in human skeletal muscle by means of $31 \mathrm{P}$ NMR. Magn. Reson. Med. 1(3). 307-315. doi:10.1002/mrm.1910010303. PMID:6571561. Boyer, K.A., Andriacchi, T.P., and Beaupre, G.S. 2012. The role of physical activity in changes in walking mechanics with age. Gait Posture. 36(1). 149-153.

doi:10.1016/j.gaitpost.2012.02.007. PMID:22445586.

Cappellini, G., Ivanenko, Y.P., Poppele, R.E., and Lacquaniti, F. 2006. Motor patterns in human walking and running. J. Neurophysiol. 95(6). 3426-3437. doi:10.1152/jn.00081.2006. PMID:16554517.

Chance, B., Leigh, J.S., Clark, B.J., Maris, J., Kent, J., Nioka, S., et al. 1985. Control of oxidative metabolism and oxygen delivery in human skeletal muscle: a steady-state analysis of the work/energy cost transfer function. Proc. Natl. Acad. Sci. 82(24). 8384-8388.

PMID:3866229.

Chilibeck, P.D., Paterson, D.H., McCreary, C.R., Marsh, G.D., Cunningham, D.A., and Thompson, R.T. 1998. The effects of age on kinetics of oxygen uptake and phosphocreatine in humans during exercise. Exp. Physiol. 83(01). 107-117.

doi:10.1113/expphysiol.1998.sp004087 PMID:9483424.

Christie, A., Snook, E.M., and Kent-Braun, J.A. 2011. Systematic review and meta-analysis of skeletal muscle fatigue in old age. Med. Sci. Sports. Exerc. 43(4). 568.

doi:10.1249/mss.0b013e3181f9b1c4 PMID:20881888.

Christie, A.D., Tonson, A., Larsen, R.G., DeBlois, J.P., and Kent, J.A. 2014. Human skeletal muscle metabolic economy in vivo: effects of contraction intensity, age, and mobility impairment. Am. J. Physiol. Regul. Integr Comp. Physiol. 307(9). R1124-R1135.

doi:10.1152/ajpregu.00083.2014. PMID:25163917. 
Coggan, A.R., Spina, R.J., Rogers, M.A., King, D.S., Brown, M., Nemeth, P.M., et al. 1990. Histochemical and enzymatic characteristics of skeletal muscle in master athletes. J. Appl. Physiol. 68(5). 1896-1901. PMID:2361892.

Colley, R.C., Garriguet, D., Janssen, I., Craig, C.L., Clarke, J., and Tremblay, M.S. 2011. Physical activity of Canadian adults: Accelerometer results from the 2007 to 2009 Canadian Health Measures Survey. Health Rep. 22(1). doi:10.1186/1471-2458-13-200 PMID:21510585.

Conley, K.E., Jubrias, S.A., and Esselman, P.C. 2000. Oxidative capacity and ageing in human muscle. J. Physiol. 526(1). 203-210. doi:10.1111/j.1469-7793.2000.t01-1-00203.x PMID:10878112.

DeVita, P. and Hortobagyi, T. 2000. Age causes a redistribution of joint torques and powers during gait. J. Appl. Physiol. 88(5). 1804-1811. PMID:10797145.

Egger, M., Smith, G.D., Schneider, M., and Minder, C. 1997. Bias in meta-analysis detected by a simple, graphical test. Br. Med. J. 315(7109). 629-634. doi:10.1136/bmj.315.7109.629 PMID:9310563.

Gouspillou, G., Sgarioto, N., Kapchinsky, S., Purves-Smith, F., Norris, B., Pion, C.H., et al. 2014. Increased sensitivity to mitochondrial permeability transition and myonuclear translocation of endonuclease $\mathrm{G}$ in atrophied muscle of physically active older humans. FASEB J. 28(4). 1621-1633. doi:10.1096/fj.13-242750. PMID:24371120.

Hart, C.R., Layec, G., Trinity, J.D., Liu, X., Kim, S.E., Groot, H.J., et al. 2014. Evidence of preserved oxidative capacity and oxygen delivery in the plantar flexor muscles with age. J. Gerontol. A Biol. Sci. Med. Sci. 1067-1076. doi:10.1093/gerona/glu139. PMID:25165028. Haseler, L.J., Hogan, M.C., and Richardson, R.S. 1999. Skeletal muscle phosphocreatine recovery in exercise-trained humans is dependent on $\mathrm{O} 2$ availability. J. Appl. Physiol. 86(6). 2013-2018. PMID:10368368. 
Haseler, L.J., Lin, A.P., and Richardson, R.S. 2004. Skeletal muscle oxidative metabolism in sedentary humans: 31P-MRS assessment of O2 supply and demand limitations. J. Appl. Physiol. 97(3). 1077-1081. doi:10.1152/japplphysiol.01321.2003. PMID:15133010. Hedges, L.V. 1984. Advances in statistical methods for meta-analysis.In: W.H. Yeaton and P.M. Wortman (Eds.), Issues in data synthesis: New directions for program evaluation, pp. 25-42. San Francisco, CA: Academic Press.

Hepple, R.T. 2014. Mitochondrial involvement and impact in aging skeletal muscle. Front Aging Neurosci 6(211). 1-13. doi:10.3389/fnagi.2014.00211 PMID:25309422.

Higgins, J.P., Thompson, S.G., Deeks, J.J., and Altman, D.G. 2003. Measuring inconsistency in meta-analyses. Br. Med. J. 327(7414). 557-560. doi:10.1136/bmj.327.7414.557. PMID:12958120.

Horská, A., Fishbein, K.W., Fleg, J.L., and Spencer, R.G.S. 2000. The relationship between creatine kinase kinetics and exercise intensity in human forearm is unchanged by age. Am. J. Physiol. Endocrinol. Metab. 279(2). E333-E339. PMID:10913033.

Hütter, E., Skovbro, M., Lener, B., Prats, C., Rabøl, R., Dela, F., et al. 2007. Oxidative stress and mitochondrial impairment can be separated from lipofuscin accumulation in aged human skeletal muscle. Aging Cell 6(2). 245-256. doi:10.1111/j.1474-9726.2007.00282.x. PMID:17376148.

Jakobsson, F., Borg, K., and Edström, L. 1990. Fibre-type composition, structure and cytoskeletal protein location of fibres in anterior tibial muscle. Acta Neuropath. 80(5). 459468. PMID:2251902.

Johannsen, D.L., Conley, K.E., Bajpeyi, S., Punyanitya, M., Gallagher, D., Zhang, Z., et al. 2011. Ectopic lipid accumulation and reduced glucose tolerance in elderly adults are accompanied by altered skeletal muscle mitochondrial activity. J. Clin. Endocrinol. Metab. 97(1). 242-250. doi:10.1210/jc.2011-1798. PMID:22049170. 
Kent-Braun, J.A., Miller, R.G., and Weiner, M.W. 1995. Human skeletal muscle metabolism in health and disease: utility of magnetic resonance spectroscopy. Exerc. Sport. Sci. Rev. 23(1). 305-348. PMID:7556355.

Kent-Braun, J.A. and Ng, A.V. 2000. Skeletal muscle oxidative capacity in young and older women and men. J. Appl. Physiol. 89(3). 1072-1078. PMID:10956353.

Kutsuzawa, T.O., Shioya, S.U., Kurita, D.A., Haida, M.U., and Yamabayashi, H.A. 2001. Effects of age on muscle energy metabolism and oxygenation in the forearm muscles. Med. Sci. Sports. Exerc. 33(6). 901-906. doi:10.1097/00005768-200106000-00008. PMID:11404654.

Lanza, I.R., Befroy, D.E., and Kent-Braun, J.A. 2005. Age-related changes in ATP-producing pathways in human skeletal muscle in vivo. J. Appl. Physiol. 99(5). 1736-1744. doi:10.1152/japplphysiol.00566.2005. PMID:16002769. Lanza, I.R., Bhagra, S., Nair, K.S., and Port, J.D. 2011. Measurement of human skeletal muscle oxidative capacity by $31 \mathrm{P}-\mathrm{MR}$ spectroscopy: A cross-validation with in vitro measurements. J. Magn. Reson. Imaging. 34(5). 1143-1150. doi:10.1002/jmri.22733. PMID:22006551.

Lanza, I.R., Larsen, R.G., and Kent-Braun, J.A. 2007. Effects of old age on human skeletal muscle energetics during fatiguing contractions with and without blood flow. J. Physiol. 583(3). 1093-1105. doi:10.1113/jphysiol.2007.138362. PMID:17673506.

Larsen, R.G., Callahan, D.M., Foulis, S.A., and Kent-Braun, J.A. 2012. Age-related changes in oxidative capacity differ between locomotory muscles and are associated with physical activity behavior. Appl. Physiol. Nutr. Metab. 37(1). 88-99. PMID:22236246. Larson-Meyer, D.E., Newcomer, B.R., Hunter, G.R., Hetherington, H.P., and Weinsier, R.L. 2000. 31P MRS measurement of mitochondrial function in skeletal muscle: reliability, force- 
level sensitivity and relation to whole body maximal oxygen uptake. NMR. Biomed. 13(1). 14-27. PMID:10668050.

Larson-Meyer, D.E., Newcomer, B.R., Hunter, G.R., Joanisse, D.R., Weinsier, R.L., and Bamman, M.M. 2001. Relation between in vivo and in vitro measurements of skeletal muscle oxidative metabolism. Muscle Nerve. 24(12). 1665-1676. doi:10.1002/mus.1202.

PMID:11745976.

Layec, G., Bringard, A., Le Fur, Y., Vilmen, C., Micallef, J.P., Perrey, S., et al. 2009.

Reproducibility assessment of metabolic variables characterizing muscle energetics in Vivo:

A 31P-MRS study. Magn. Reson. Med. 62(4). 840-854. doi:10.1002/mrm.22085.

PMID:19725136.

Layec, G., Hart, C.R., Trinity, J.D., Le Fur, Y., Jeong, E.K., and Richardson, R.S. 2015.

Skeletal muscle work efficiency with age: the role of non-contractile processes. Clin. Sci.

128(3). 213-223. doi:10.1042/cs20140274. PMID:25134525.

Layec, G., Haseler, L.J., and Richardson, R.S. 2013. Reduced muscle oxidative capacity is independent of O2 availability in elderly people. Age. 35(4). 1183-1192. PMID:22760857. Lexell, J., Taylor, C.C., and Sjöström, M. 1988. What is the cause of the ageing atrophy?: Total number, size and proportion of different fiber types studied in whole vastus lateralis muscle from 15-to 83-year-old men. J. Neurol. Sci. 84(2). 275-294. PMID:3379447. Marsh, G.D., Paterson, D.H., Potwarka, J.J., and Thompson, R.T. 1993. Transient changes in muscle high-energy phosphates during moderate exercise. J. Appl. Physiol. 75(2). 648-656. PMID:8226464.

Mattei, J.P., Bendahan, D., Roussel, M., Lefur, Y., and Cozzone, P.J. 1999. Gender modulates the energy cost of muscle contraction in untrained healthy subjects. A $31 \mathrm{P}$ magnetic resonance spectroscopy analysis. FEBS letters 450(3). 173-177. 
McCully, K.K., Fielding, R.A., Evans, W.J., Leigh, J.S., and Posner, J.D. 1993. Relationships between in vivo and in vitro measurements of metabolism in young and old human calf muscles. J. Appl. Physiol. 75(2). 813-819. PMID:8226486.

Meyer, R.A. 1988. A linear model of muscle respiration explains monoexponential phosphocreatine changes. Am. J. Physiol. Cell. Physiol. 254(4). C548-C553. PMID:3354652. Paganini, A.T., Foley, J.M., and Meyer, R.A. 1997. Linear dependence of muscle phosphocreatine kinetics on oxidative capacity. Am. J. Physiol. Cell. Physiol. 272(2). C501C510. PMID:9124293.

Picard, M., Ritchie, D., Thomas, M.M., Wright, K.J., and Hepple, R.T. 2011a. Alterations in intrinsic mitochondrial function with aging are fiber type-specific and do not explain differential atrophy between muscles. Aging Cell 10(6). 1047-1055. PMID:21933339.

Picard, M., Taivassalo, T., Gouspillou, G., and Hepple, R.T. 2011b. Mitochondria: isolation, structure and function. J. Physiol. 589(18). 4413-4421. doi:10.1113/jphysiol.2011.212712. PMID:21708903.

Picard, M., Taivassalo, T., Ritchie, D., Wright, K.J., Thomas, M.M., Romestaing, C., et al. 2011c. Mitochondrial structure and function are disrupted by standard isolation methods. PloS one 6(3). e18317. doi:10.1371/journal.pone.0018317. PMID:21512578.

Proctor, D.N. and Joyner, M.J. 1997. Skeletal muscle mass and the reduction of VO2max in trained older subjects. J. Appl. Physiol. 82(5). 1411-1415. PMID:9134886.

Quistorff, B., Johansen, L., and Sahlin, K. 1993. Absence of phosphocreatine resynthesis in human calf muscle during ischaemic recovery. Biochem J. 291(3). 681-686.

doi:10.1042/bj2910681. PMID:8489495.

Russ, D.W. and Kent-Braun, J.A. 2004. Is skeletal muscle oxidative capacity decreased in old age? Sports Med. 34(4). 221-229. PMID:15049714. 
Sahlin, K. and Harris, R.C. 2011. The creatine kinase reaction: a simple reaction with functional complexity. Amino Acids 40(5). 1363-1367. doi:10.1007/s00726-011-0856-8. PMID:21394603.

Saks, V.A., Veksler, V.I., Kuznetsov, A.V., Kay, L., Sikk, P., Tiivel, T., et al. 1998.

Permeabilized cell and skinned fiber techniques in studies of mitochondrial function in vitro. Mol Cell Biochem 184(1-2). 81-100. PMID:9746314.

Short, K.R., Bigelow, M.L., Kahl, J., Singh, R., Coenen-Schimke, J., Raghavakaimal, S., et al. 2005. Decline in skeletal muscle mitochondrial function with aging in humans. Proc. Natl. Acad. Sci. USA 102(15). 5618-5623. doi:doi: 10.1073/pnas.0501559102. PMID:15800038. Smith, S.A., Montain, S.J., Matott, R.P., Zientara, G.P., Jolesz, F.A., and Fielding, R.A. 1998. Creatine supplementation and age influence muscle metabolism during exercise. J. Appl. Physiol. 85(4). 1349-1356. PMID:9760327.

Strath, S.J., Kaminsky, L.A., Ainsworth, B.E., Ekelund, U., Freedson, P.S., Gary, R.A., et al. 2013. Guide to the assessment of physical activity: Clinical and research applications: a scientific statement from the American Heart Association. Circulation 128(20). 2259-79. doi:10.1161/01.cir.0000435708.67487.da. PMID:24126387.

Stroup, D.F., Berlin, J.A., Morton, S.C., Olkin, I., Williamson, G.D., Rennie, D., et al. 2000. Meta-analysis of observational studies in epidemiology: a proposal for reporting. JAMA. 283(15). 2008-2012. doi:10.1001/jama.283.15.2008. PMID:10789670.

Sutton, A.J. and Higgins, J. 2008. Recent developments in meta-analysis. Stat. Med. 27(5). 625-650. PMID:17590884.

Taylor, D.J., Crowe, M., Bore, P.J., Styles, P., Arnold, D.L., and Radda, G.K. 1984. Examination of the energetics of aging skeletal muscle using nuclear magnetic resonance. Gerontology. 30(1). 2-7. doi:10.1159/000212599. PMID:6698405. 
Taylor, D.J., Kemp, G.J., Thompson, C.H., and Radda, G.K. 1997. Ageing: effects on oxidative function of skeletal muscle in vivo. Mol. Cell. Biochem. 174(1-2). 321-324. PMID:9309705.

Tevald, M.A., Foulis, S.A., and Kent, J.A. 2014. Effect of age on in vivo oxidative capacity in two locomotory muscles of the leg. Age. 36(5). 1-13. doi:10.1007/s11357-014-9713-5. PMID:25227177.

Tevald, M.A., Foulis, S.A., Lanza, I.R., and Kent-Braun, J.A. 2010. Lower energy cost of skeletal muscle contractions in older humans. Am. J. Physiol. Regul. Integr Comp. Physiol. 298(3). R729-R739. doi:10.1152/ajpregu.00713.2009. PMID:20032262.

Tonkonogi, M., Fernström, M., Walsh, B., Ji, L.L., Rooyackers, O., Hammarqvist, F., et al. 2003. Reduced oxidative power but unchanged antioxidative capacity in skeletal muscle from aged humans. Pflügers Archiv. 446(2). 261-269. doi:10.1007/s00424-003-1044-9.

PMID:12684796.

Troiano, R.P., Berrigan, D., Dodd, K.W., Masse, L.C., Tilert, T., and McDowell, M. 2008. Physical activity in the United States measured by accelerometer. Med. Sci. Sports. Exerc. 40(1). 181-8. doi:10.1249/mss.0b013e31815a51b3. PMID:18091006.

Walsh, B., Tonkonogi, M., Söderlund, K., Hultman, E., Saks, V., and Sahlin, K. 2001. The role of phosphorylcreatine and creatine in the regulation of mitochondrial respiration in human skeletal muscle. J. Physiol. 537(3). 971-978. PMID:2278998.

Waters, D.L., Brooks, W.M., Qualls, C.R., and Baumgartner, R.N. 2003. Skeletal muscle mitochondrial function and lean body mass in healthy exercising elderly. Mech. Ageing Dev. 124(3). 301-309. doi:10.1016/s0047-6374(02)00197-5. PMID:12663127.

Wray, D.W., Nishiyama, S.K., Monnet, A., Wary, C., Duteil, S., Carlier, P.G., et al. 2009. Multiparametric NMR-based assessment of skeletal muscle perfusion and metabolism during 
exercise in elderly persons: preliminary findings. J. Gerontol. A Biol. Sci. Med. Sci. 64(9). 968-974. doi:10.1093/gerona/glp044. PMID:19377015. 
Table 1. Distribution of effects by age, for each moderator

\begin{tabular}{|c|c|c|c|}
\hline Oxidative Capacity: & Older $>$ Young & Older $=$ Young & Older $<$ Young \\
\hline \# Effects (\# Studies) & $14(12)$ & $1(1)$ & $13(12)$ \\
\hline \multicolumn{4}{|l|}{ Age (mean \pm SD) } \\
\hline Young & $26.3 \pm 4.0$ & $26 \pm 3.8$ & $28.4 \pm 4.2$ \\
\hline Older & $71.1 \pm 4.0$ & $72 \pm 4.6$ & $70.4 \pm 5.0$ \\
\hline \multicolumn{4}{|l|}{ Moderators } \\
\hline \multicolumn{4}{|l|}{ Muscle Group } \\
\hline $\mathrm{KE}$ & $0(0)$ & $0(0)$ & $5(5)$ \\
\hline PF & $5(4)$ & $0(0)$ & $6(5)$ \\
\hline UE & $3(3)$ & $0(0)$ & $1(1)$ \\
\hline DF & $6(6)$ & $1(1)$ & $1(1)$ \\
\hline \multicolumn{4}{|l|}{ Physical Activity } \\
\hline High & $1(1)$ & $0(0)$ & $1(1)$ \\
\hline Moderate & $1(1)$ & $0(0)$ & $1(1)$ \\
\hline Low & $8(7)$ & $1(1)$ & $6(6)$ \\
\hline Not reported & $4(4)$ & $0(0)$ & $5(5)$ \\
\hline \multicolumn{4}{|l|}{$\operatorname{Sex}$} \\
\hline Mixed & $10(9)$ & $0(0)$ & $8(8)$ \\
\hline Female & $0(0)$ & $0(0)$ & $3(2)$ \\
\hline Male & $4(3)$ & $1(1)$ & $1(1)$ \\
\hline \multicolumn{4}{|c|}{ PCr Depletion (\% baseline) } \\
\hline No difference & $7(6)$ & $1(1)$ & $6(6)$ \\
\hline$>$ in older & $1(1)$ & $0(0)$ & $2(2)$ \\
\hline$>$ in younger & $2(2)$ & $0(0)$ & $1(1)$ \\
\hline Not reported & $4(3)$ & $0(0)$ & $4(3)$ \\
\hline \multicolumn{4}{|l|}{ End-contraction $\mathrm{pH}$} \\
\hline No difference & $9(8)$ & $0(0)$ & $6(6)$ \\
\hline$>$ in older & $3(3)$ & $1(1)$ & $3(3)$ \\
\hline$>$ in younger & $0(0)$ & $0(0)$ & $1(1)$ \\
\hline Not reported & $2(1)$ & $0(0)$ & $3(2)$ \\
\hline
\end{tabular}

Data are mean \pm SD for age, and the number of effects (and number of studies) for the mean effect and those for each moderator. A positive effect indicates older $>$ young; a negative effect indicates young > older. UE, upper extremity; PF, plantar flexors; DF, dorsiflexors; $\mathrm{KE}$, knee extensors; PCr, phosphocreatine. 
Table 2. Impact of muscle group on age-related differences in muscle oxidative capacity

\begin{tabular}{|c|c|c|c|c|c|}
\hline $\begin{array}{c}\text { Study } \\
\text { (by publication date) }\end{array}$ & $\begin{array}{l}\text { Muscle } \\
\text { Group }\end{array}$ & Sex & $\begin{array}{l}\text { Physical } \\
\text { Activity }\end{array}$ & $\begin{array}{l}\text { Hedges' } \\
\quad g\end{array}$ & $\begin{array}{l}\text { Standard } \\
\text { error }\end{array}$ \\
\hline Smith et al. 1998 & $\mathrm{KE}$ & $\mathrm{M}, \mathrm{W}$ & $\mathrm{N} / \mathrm{A}$ & -0.755 & 0.643 \\
\hline Conley et al. 2000 & $\mathrm{KE}$ & $\mathrm{M}, \mathrm{W}$ & $\mathrm{N} / \mathrm{A}$ & $-7.649 *$ & 0.854 \\
\hline Johannsen et al. 2011 & $\mathrm{KE}$ & $\mathrm{M}, \mathrm{W}$ & Low & $-2.779 *$ & 0.513 \\
\hline Larsen et al. 2012 & $\mathrm{KE}$ & $\mathrm{M}, \mathrm{W}$ & Low & $-0.689 *$ & 0.355 \\
\hline Layec et al. 2015 & $\mathrm{KE}$ & $\mathrm{M}, \mathrm{W}$ & Low & -0.289 & 0.441 \\
\hline$K E(m e a n \pm S D)$ & & & & $-1.444^{*}$ & 0.220 \\
\hline McCully et al. 1993 & $\mathrm{PF}$ & $\mathrm{M}, \mathrm{W}$ & $\mathrm{N} / \mathrm{A}$ & $-1.335^{*}$ & 0.655 \\
\hline Taylor et al. 1997 & $\mathrm{PF}$ & $\mathrm{M}, \mathrm{W}$ & $\mathrm{N} / \mathrm{A}$ & -0.582 & 0.437 \\
\hline Chilibeck et al. 1998 & $\mathrm{PF}$ & $\mathrm{M}, \mathrm{W}$ & N/A & 0.221 & 0.430 \\
\hline Waters et al. 2003 & $\mathrm{PF}$ & M & High & $1.374 *$ & 0.573 \\
\hline Waters et al. 2003 & $\mathrm{PF}$ & $\mathrm{W}$ & High & $-1.167 *$ & 0.538 \\
\hline Waters et al. 2003 & $\mathrm{PF}$ & M & Low & 0.737 & 0.521 \\
\hline Waters et al. 2003 & $\mathrm{PF}$ & $\mathrm{W}$ & Low & $-3.008 *$ & 0.753 \\
\hline Wray et al. 2009 & $\mathrm{PF}$ & $\mathrm{M}, \mathrm{W}$ & Moderate & -0.213 & 0.535 \\
\hline Layec et al. 2013 & $\mathrm{PF}$ & M & Low & $-1.409 *$ & 0.606 \\
\hline Tevald et al. 2014 & $\mathrm{PF}$ & $\mathrm{M}, \mathrm{W}$ & Low & 0.574 & 0.342 \\
\hline Hart et al. 2014 & $\mathrm{PF}$ & $\mathrm{M}, \mathrm{W}$ & Moderate & 0.214 & 0.311 \\
\hline$P F(m e a n \pm S D)$ & & & & -0.097 & 0.141 \\
\hline Taylor et al. 1984 & $\overline{\mathrm{UE}}$ & $\mathrm{M}, \mathrm{W}$ & $\mathrm{N} / \mathrm{A}$ & -0.303 & 0.342 \\
\hline Mattei et al. 1999 & UE & $\mathrm{M}, \mathrm{W}$ & $\mathrm{N} / \mathrm{A}$ & $1.004 *$ & 0.262 \\
\hline Horská et al. 2000 & UE & $\mathrm{M}, \mathrm{W}$ & $\mathrm{N} / \mathrm{A}$ & 0.215 & 0.367 \\
\hline Kutsuzawa et al. 2001 & UE & $\mathrm{M}, \mathrm{W}$ & $\mathrm{N} / \mathrm{A}$ & 0.367 & 0.453 \\
\hline$U E(\operatorname{mean} \pm S D)$ & & & & $0.434 *$ & 0.168 \\
\hline Kent-Braun and Ng 2000 & $\overline{\mathrm{DF}}$ & $\mathrm{M}$ & Low & $1.166^{*}$ & 0.349 \\
\hline Kent-Braun and Ng 2000 & $\mathrm{DF}$ & $\mathrm{W}$ & Low & -0.422 & 0.326 \\
\hline Lanza et al. 2005 & DF & M & Low & 0.742 & 0.491 \\
\hline Lanza et al. 2007 & $\mathrm{DF}$ & $\mathrm{M}, \mathrm{W}$ & Low & $2.436^{*}$ & 0.423 \\
\hline Tevald et al. 2010 & DF & M & Low & 0.000 & 0.449 \\
\hline Larsen et al. 2012 & $\mathrm{DF}$ & $\mathrm{M}, \mathrm{W}$ & Low & $0.827 *$ & 0.360 \\
\hline Christie et al. 2014 & DF & $\mathrm{M}, \mathrm{W}$ & Low & $0.979 *$ & 0.337 \\
\hline Tevald et al. 2014 & DF & $\mathrm{M}, \mathrm{W}$ & Low & $1.381 *$ & 0.374 \\
\hline$D F(\operatorname{mean} \pm S D)$ & & & & $0.841 *$ & 0.134 \\
\hline Overall Mean & & & & $0.171 *$ & 0.079 \\
\hline
\end{tabular}

The effect (Hedges' g) and standard error of the effect for each study. Studies are sorted by muscle group. A description of the sex and physical activity status of participants within a study is provided. * indicates a significant difference between young and older muscle at the $\mathrm{p} \leq 0.05$ level. KE, knee extensors; PF, plantar flexors; UE, upper extremity; DF, dorsiflexors;

$446 \mathrm{M}$, men; $\mathrm{W}$, women. 
Table 3. Effects and heterogeneity for each sub-group within the moderators

\begin{tabular}{|c|c|c|c|c|c|c|}
\hline Moderator & Sub-group & $\mathbf{n}$ & $\begin{array}{c}\text { Hedges' } \\
\text { g }\end{array}$ & $95 \% \mathrm{CI}$ & $\begin{array}{c}\begin{array}{c}\text { P-value } \\
\text { for }\end{array} \\
\text { Q-statistic }\end{array}$ & $\begin{array}{c}I^{2} \\
(\%)\end{array}$ \\
\hline \multicolumn{7}{|l|}{ Muscle Group } \\
\hline & $\mathrm{KE}$ & 5 & -1.44 & -1.88 to -1.01 & 0.000 & 94.5 \\
\hline & $\mathrm{PF}$ & 11 & -0.10 & -0.37 to 0.18 & 0.000 & 76.7 \\
\hline & UE & 4 & 0.43 & 0.11 to 0.76 & 0.021 & 69.3 \\
\hline & DF & 8 & 0.84 & 0.60 to 1.10 & 0.000 & 80.5 \\
\hline \multicolumn{7}{|l|}{ Physical Activity } \\
\hline & High & 2 & 0.02 & -0.75 to 0.79 & 0.001 & 90.4 \\
\hline & Moderate & 2 & 0.11 & -0.42 to 0.63 & 0.490 & 0.0 \\
\hline & Low & 15 & 0.31 & 0.11 to 0.52 & 0.000 & 88.9 \\
\hline \multicolumn{7}{|l|}{$\operatorname{Sex}$} \\
\hline & Mixed & 18 & 0.25 & 0.07 to 0.44 & 0.000 & 91.1 \\
\hline & Female & 3 & -0.93 & -1.46 to -0.40 & 0.005 & 81.0 \\
\hline & Male & 6 & 0.62 & 0.23 to 1.02 & 0.004 & 70.7 \\
\hline \multirow{2}{*}{\multicolumn{7}{|c|}{ PCr Depletion }} \\
\hline & & & & & & \\
\hline & No difference & 14 & 0.17 & -0.04 to 0.37 & 0.000 & 70.7 \\
\hline & $>$ in older & 3 & 0.17 & -0.27 to 0.62 & 0.000 & 97.9 \\
\hline & $>$ in younger & 3 & 1.32 & 0.83 to 1.80 & 0.000 & 88.6 \\
\hline \multicolumn{7}{|c|}{ End-Contraction $p H$} \\
\hline & No difference & 15 & 0.46 & 0.26 to 0.66 & 0.000 & 81.1 \\
\hline & $>$ in older & 7 & 0.13 & -0.15 to 0.42 & 0.010 & 64.6 \\
\hline & $>$ in younger & 1 & -7.65 & -9.32 to -5.98 & 1.000 & 0.00 \\
\hline
\end{tabular}


452

453 Figure 1. Funnel plot of standard error and effect for each study. The 28 effects and their

454 associated SE (from Table 2) are represented by open circles. The angled lines represent the area in which $95 \%$ of the effects are expected to lie, in the absence of publication bias. The straight vertical line signifies the middle of the funnel, and equal numbers of effects about this 457 line indicate the absence of publication bias.

458

459

Figure 2. Overall and sub-group standardized effects (Hedges' g) and SE for each

460

moderator. The shaded bars illustrate the overall effect of each moderator along with the standard error of estimate. The open bars show the effect for sub-groups of the moderators, 462 along with the standard error of estimate. *indicates a significant effect of that moderator or 463 moderator sub-group on muscle oxidative capacity at the $\mathrm{p}<0.05$ level. Positive and negative 464 effects indicate greater and lower oxidative capacity in older adults compared with young 465 adults, respectively. 
0.0

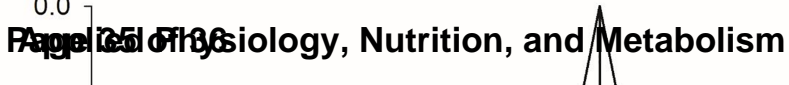

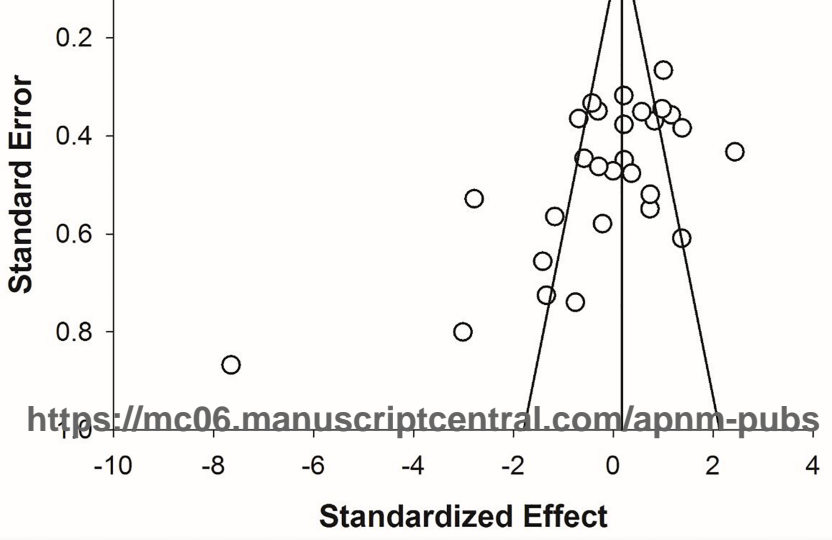




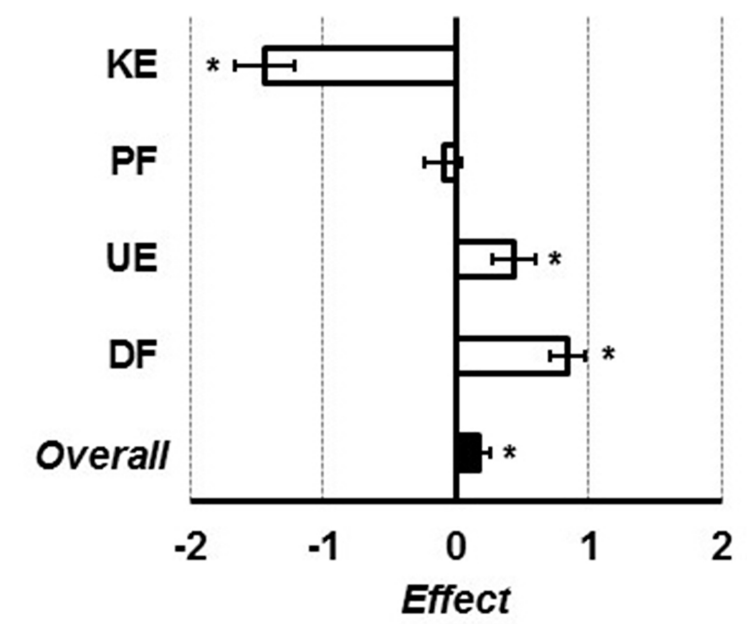

Physical Activity

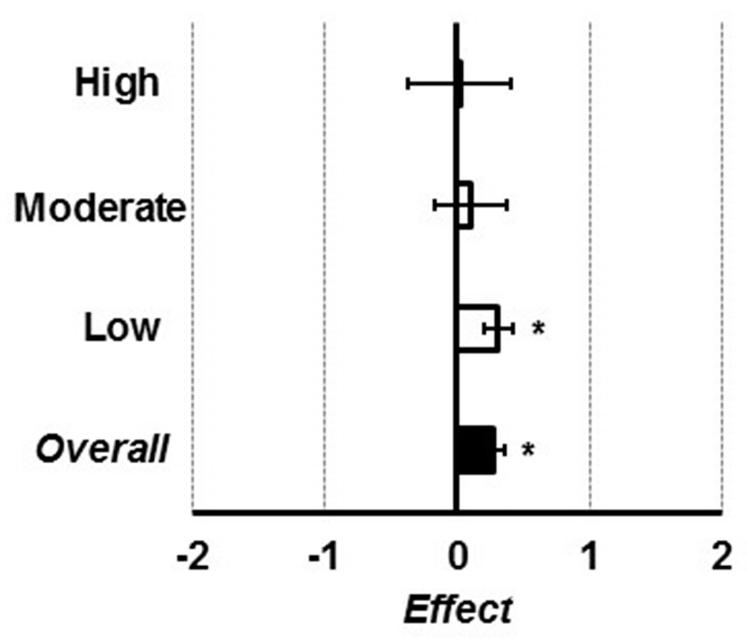

Sex

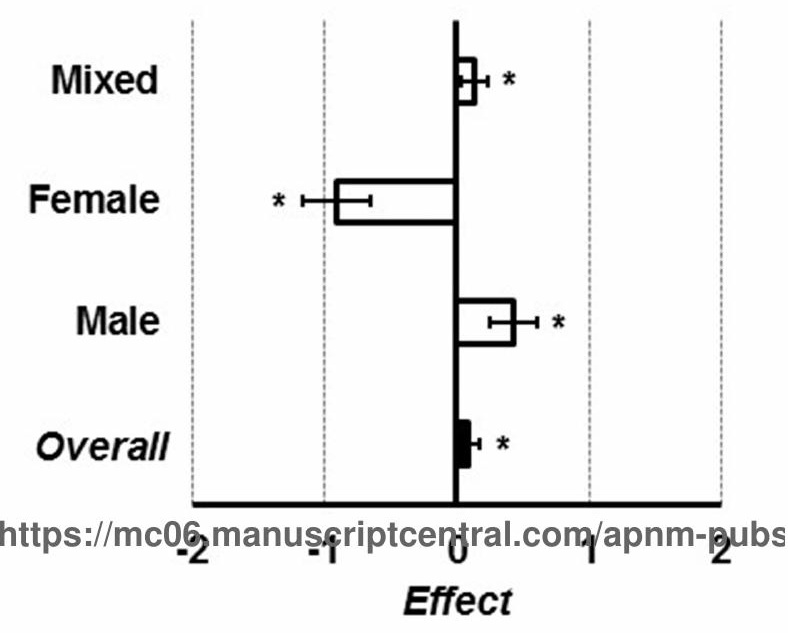

